\title{
Reliability Evaluation of Bridges Based on Nonprobabilistic Response Surface Limit Method
}

\author{
Xuyong Chen,, Qian Chen, ${ }^{1}$ Xiaoya Bian, ${ }^{1}$ and Jianping Fan ${ }^{2}$ \\ ${ }^{1}$ School of Resource and Civil Engineering, Wuhan Institute of Technology, Wuhan 430073, China \\ ${ }^{2}$ School of Civil Engineering \& Mechanics, Huazhong University of Science and Technology, Wuhan 430074, China \\ Correspondence should be addressed to Xiaoya Bian; bianxy@hust.edu.cn
}

Received 14 October 2017; Accepted 10 December 2017; Published 28 December 2017

Academic Editor: Xiangyu Meng

Copyright (c) 2017 Xuyong Chen et al. This is an open access article distributed under the Creative Commons Attribution License, which permits unrestricted use, distribution, and reproduction in any medium, provided the original work is properly cited.

\begin{abstract}
Due to many uncertainties in nonprobabilistic reliability assessment of bridges, the limit state function is generally unknown. The traditional nonprobabilistic response surface method is a lengthy and oscillating iteration process and leads to difficultly solving the nonprobabilistic reliability index. This article proposes a nonprobabilistic response surface limit method based on the interval model. The intention of this method is to solve the upper and lower limits of the nonprobabilistic reliability index and to narrow the range of the nonprobabilistic reliability index. If the range of the reliability index reduces to an acceptable accuracy, the solution will be considered convergent, and the nonprobabilistic reliability index will be obtained. The case study indicates that using the proposed method can avoid oscillating iteration process, make iteration process stable and convergent, reduce iteration steps significantly, and improve computational efficiency and precision significantly compared with the traditional nonprobabilistic response surface method. Finally, the nonprobabilistic reliability evaluation process of bridge will be built through evaluating the reliability of one PC continuous rigid frame bridge with three spans using the proposed method, which appears to be more simple and reliable when lack of samples and parameters in the bridge nonprobabilistic reliability evaluation is present.
\end{abstract}

\section{Introduction}

There are many unavoidable uncertainties in practical structure engineering. Traditionally, the probability model is utilized in the structural reliability analysis [1]. Probabilistic reliability analysis strongly depends on the probability distribution function, which relies on a large number of statistical data [2]. However, for some important and complicated structures, many uncertain parameters have little or no statistical data, which causes difficulties in accurate description of parameter distribution. In addition, probabilistic reliability is very sensitive to variations of model parameters. Small errors in statistical data can lead to considerable errors in the structure $[2,3]$.

Because of inadequate data, the probabilistic reliability is not useful for solving these practical problems. However, nonprobabilistic reliability can effectively deal with reliability problems when only few statistical data can be obtained. A nonprobabilistic convex model was first proposed in the 1990s by Ben-Haim [4, 5]. Ben-Haim and Elishakoff [6] proposed a nonprobabilistic safety factor to measure the nonprobabilistic reliability index using an interval theory. The nonprobabilistic reliability theories presented by Ben-Haim $[4,5]$ and Ben-Haim and Elishakoff [6] were not involved in probability at all and could overcome the inextricable difficulties faced by the traditional probability model. Therefore, nonprobabilistic reliability is an appropriate method when the available data of uncertainties are limited or absent. This was illustrated by Guo et al. [7, 8], who contrasted the probabilistic and nonprobabilistic reliability methods through modeling concepts, model construction, and formulations for computation. Nonprobabilistic reliability of structures has become a new, exciting research direction, and the corresponding research approach has also aroused wide attention from theory and engineering circles.

In summary, nonprobabilistic reliability analysis was generally based on the interval model $[9,10]$ or the convex model [11-13]. The nonprobabilistic reliability index based on the interval model is actually the minimum norm of the coordinate vector in the standardized space, and solving the 
reliability index is an optimum problem with the equality constraint. For the linear performance functions, the analytical expression of the nonprobabilistic reliability index can be easily obtained. However, the performance functions are generally nonlinear in practical engineering.

For simple nonlinear performance functions, Guo et al. $[7,8]$ suggested definition approach, transfer approach, and optimization approach to solve the nonprobabilistic reliability index. For complexly and strongly nonlinear performance functions, researchers frequently used an optimized iterative algorithm. For the hyperellipsoidal model, the nonprobabilistic reliability index is in accordance with the probabilistic reliability index in definition, so the design point method, successfully applied to the probabilistic reliability analysis, can be used for the nonprobabilistic reliability analysis [14]. For the reliability index defined by the Euclidean norm, the Most Probable failure Point (MPP) can be obtained along the normal direction of the limit state surface. For the nonprobabilistic reliability index based on the interval model defined by the infinite norm, the MPP might not be along the normal direction of the limit state surface.

In order to simplify the search process, other researchers suggested the one-dimensional optimization method $[15,16]$ and the space search algorithm [17]. These two methods are correct only for the linear performance function, because only part of the probable failure points is searched for the nonlinear performance function. If the performance functions are the normalized quadratic expression, the Sequence Quadratic Programming (SQP) can be used to solve the nonprobabilistic reliability index based on the interval model [18]. Recently, the Gradient Projection Method (GPM) was proposed to solve the nonprobabilistic reliability index [19]. GPM is the general method that is most suitable for the nonprobabilistic convex model, although the convergence process during the iteration needs special treatment. The interval model was used in the structural reliability optimization design $[20,21]$, although this issue is not discussed in this article in detail.

When the limit state function is not easily obtained, the above methods are not applicable. Jiang et al. [22] presented the nonprobabilistic response surface method based on the interval model, which contributed to solving the nonprobabilistic reliability index for the implicit performance function. Chen et al. [23] used the response surface method to build the explicit performance function, accepted the interval model and the ellipsoidal model to compute the nonprobabilistic reliability index, and compared the results from these two methods.

Until present, nonprobabilistic reliability analysis based on the convex model and the interval model has achieved some positive results and been applied in engineering practice. A great obstacle is solving the nonprobabilistic reliability index. The traditional nonprobabilistic response surface method is prone to lengthy and oscillating iteration processes, which leads to difficultly solving the nonprobabilistic reliability index. For these problems, this article will propose the nonprobabilistic response surface limit method based on the interval model and build the nonprobabilistic reliability evaluation process for bridges through evaluation of the reliability of one PC continuous rigid frame bridge with three spans.

\section{Nonprobabilistic Reliability Index Based on the Interval Model}

Suppose $\mathbf{x}=\left\{x_{1}, x_{2}, \ldots, x_{n}\right\}$ is the set of the basic interval variables for the structures, where $x_{i} \in X_{i}^{I}(i=1,2, \ldots, n)$. The performance function is given as

$$
M=g(\mathbf{x})=g\left(x_{1}, \ldots, x_{n}\right) .
$$

When $g(\mathbf{x})$ is the continuous function of $x_{i}(i=1,2$, $\ldots, n), M$ also is the interval variable. From the perspective of the nonprobabilistic reliability theory, the hypersurface $g(\mathbf{x})=0$ is the failure surface of the structures. When $g(\mathbf{x})<$ 0 , the structure is in the failure state; when $g(\mathbf{x})>0$ the structure is safe.

If $M^{c}$ and $M^{r}$ represent the mean and dispersion of $M$, respectively, the nonprobabilistic reliability index is estimated as

$$
\eta=\frac{M^{c}}{M^{r}} \in \begin{cases}{[1,+\infty)} & \text { Reliable and safe } \\ (-1,1) & \text { Unreliable } \\ (-\infty,-1] & \text { Failure. }\end{cases}
$$

Based on (2), when $\eta>1, g(\mathbf{x})>0$ for any $x_{i} \in X_{i}^{I}(i=$ $1,2, \ldots, n)$, which means the structure is in the safe state; when $\eta<-1, g(\mathbf{x})<0$ for any $x_{i} \in X_{i}^{I}(i=1,2, \ldots, n)$, which means the structure is failed; when $-1 \leq \eta \leq 1, g(\mathbf{x}) \geq 0$ and $g(\mathbf{x}) \leq 0$ maybe occurs for any $x_{i} \in X_{i}^{I}(i=1,2, \ldots, n)$, which means the structure may be safe or at risk. The greater $\eta$, the safer the structure.

Standardize the interval variable $x_{i}$ through the following transformation formula:

$$
x_{i}=x_{i}^{c}+x_{i}^{r} \delta_{i} \quad i=1,2, \ldots, n,
$$

where $x_{i}^{c}=\left(x_{i}^{l}+x_{i}^{u}\right) / 2$ and $x_{i}^{r}=\left(x_{i}^{u}-x_{i}^{l}\right) / 2$, which represent the mean and dispersion of $x_{i}$, and $x_{i}^{u}$ and $x_{i}^{l}$ are the upper and lower values of $x_{i}$, respectively. Therefore, the interval variable sets $\mathbf{x}=\left\{x_{1}, x_{2}, \ldots, x_{n}\right\}$ can be transformed into the standardized interval variable sets $\delta=\left\{\delta_{1}, \delta_{2}, \ldots, \delta_{n}\right\}$. The region of variation of the standardized interval variable $\delta_{i}$ is $[-1,1]$, and the extended range of $\delta_{i}$ is $[-\infty,+\infty]$ [15]. Substitute $\delta=\left\{\delta_{1}, \delta_{2}, \ldots, \delta_{n}\right\}$ into the failure surface $g(\mathbf{x})=0$; then the continuous performance function can be transformed into the standardized performance function:

$$
M=g\left(x_{1}, x_{2}, \ldots, x_{n}\right)=G\left(\delta_{1}, \delta_{2}, \ldots, \delta_{n}\right)=0 .
$$

In practice engineering, the performance function is generally expressed as

$$
M=g(\mathbf{x})=r-s
$$

where $r$ is the resistance and $s$ is the load. 


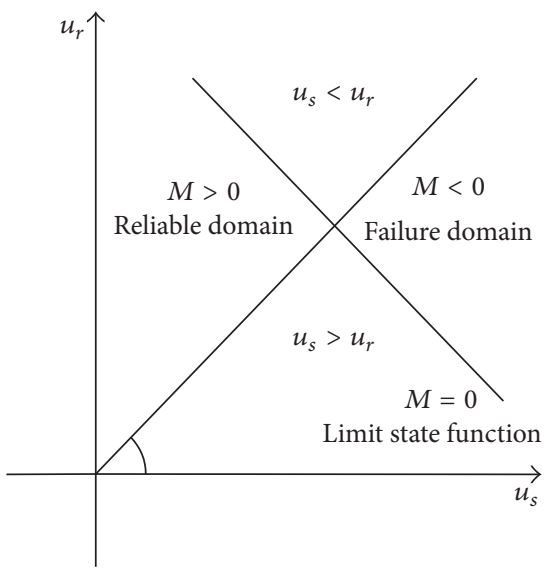

FIgURE 1: Reliability index of linear function.

(1) For the Linear Performance Function

$$
M=\sum_{i=1}^{n} a_{i} r_{i}-\sum_{j=m+1}^{n} b_{j} s_{j}=0 .
$$

Standardize $r$ and $s: r=R^{c}+R^{r} u r, s=S^{c}+S^{r} u s$, and then the linear performance function will be transformed into

$$
\begin{aligned}
M & =\sum_{i=1}^{m} a_{i} R_{i}^{r} \delta_{r_{i}}-\sum_{j=m+1}^{n} b_{i} S_{j}^{r} \delta_{s_{j}}+\left(\sum_{i=1}^{m} a_{i} R_{i}^{c}-\sum_{j=m+1}^{n} b_{i} S_{j}^{c}\right) \\
& =0
\end{aligned}
$$

and the nonprobabilistic reliability index is estimated as [24]

$\eta$

$$
= \begin{cases}\frac{\sum_{i=1}^{m} a_{i} R_{i}^{c}-\sum_{i=m+1}^{n} b_{j} S_{j}^{c}}{\sum_{i=1}^{m}\left|a_{i}\right| R_{i}^{r}-\sum_{i=m+1}^{n}\left|b_{j}\right| S_{j}^{r}}, & \sum_{i=1}^{m} a_{i} R_{i}^{c}-\sum_{i=m+1}^{n} b_{j} S_{j}^{c}>0 \\ 0, & \sum_{i=1}^{m} a_{i} R_{i}^{c}-\sum_{i=m+1}^{n} b_{j} S_{j}^{c} \leq 0\end{cases}
$$

which can be also expressed using the geometric illustration in the two-dimensional surface (Figure 1).

(2) For the Nonlinear Performance Function. The nonprobabilistic reliability index based on the interval model is defined as $[16,24]$

$$
\eta=\min \left(\|\mathbf{u}\|_{\infty}\right) .
$$

Here the interval variables $\mathbf{u}$ satisfy $G\left(u_{1}, u_{2}, \ldots, u_{n}\right)=0$, where $\|\mathbf{u}\|_{\infty}$ is the infinite norm of the vector $\mathbf{u}$; namely,

$$
\|\mathbf{u}\|_{\infty}=\max \left(\left|u_{1}\right|,\left|u_{2}\right|, \ldots,\left|u_{n}\right|\right) .
$$

In practice, the nonprobabilistic reliability index is the minimum distance from the origin of coordinates to the failure surface measured by the infinite norm in the regularized standard space. The above nonprobabilistic reliability index for the nonlinear performance function can be expressed using the geometric illustration in the two-dimensional surface (Figure 2).

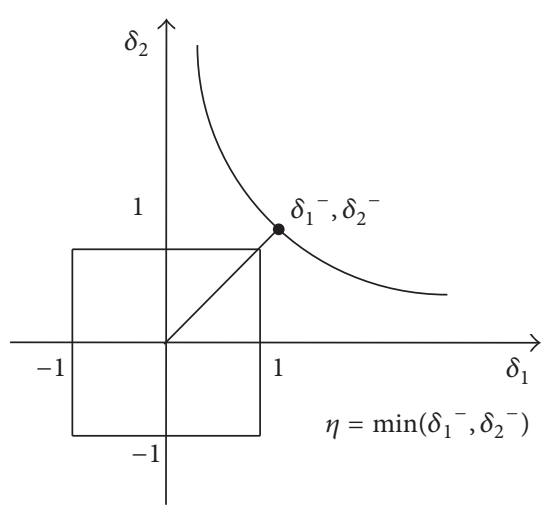

FIGURE 2: Reliability index of nonlinear function.

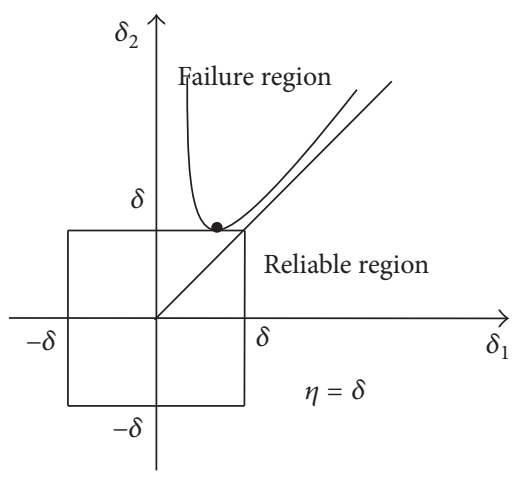

Figure 3: Most Probable failure Point above $45^{\circ}$ line.

\section{Nonprobabilistic Response Surface Limit Method}

3.1. Limit Method Basic Principle. Fan and Chen [25] pointed out that all MPPs only exist among the extreme points and the root points of the limit state function. Obviously, the tangent line must exist at the extreme points of the failure surface. Taking the two-dimensional case as an example, the square is used to approximate the performance function curve when the length of side of the square, with the origin of coordinates as its center, is increasing from 0 . With increased length of the side of the square, one side of the square will be tangent with the performance function curve, or one vertex of the square will intersect with the performance function curve. The maximum of the absolute values of coordinates for the earliest intersection is the desired nonprobabilistic reliability index.

For two-dimensional case, the MPPs may be in the region above the $45^{\circ}$ line, in the region below the $45^{\circ}$ line, or on the $45^{\circ}$ line (the boundary of these two regions). The limit method will also be discussed for these three cases, respectively (Figures 3, 4, and 5).

3.2. Response Surface Method. The response surface method was initially presented by Box and Wilson [26] and was mainly used to fit the limit state surface when the performance function was not known in practical engineering. Myers and Montgomery [27] perfected the proposed basic 


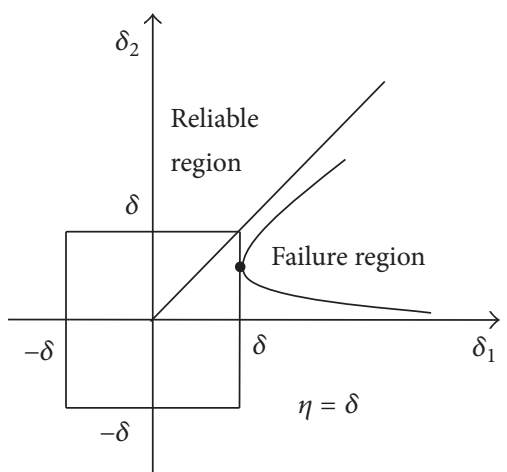

Figure 4: Most Probable failure Point below $45^{\circ}$ line.

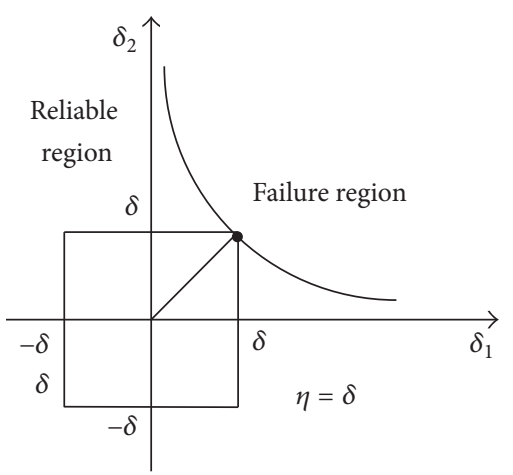

Figure 5: Most Probable failure Point on $45^{\circ}$ line.

principle of the response surface method, which was the theoretical basis for the development of the response surface method.

For linear function, the first-order response surface is generally selected to approximate the real limit state function. The established response surface function is as follows:

$$
M=g(\mathbf{x})=a+\sum_{i=1}^{n} b_{i} x_{i}+\sum_{1 \leq i \leq j \leq n} c_{i j} x_{i} x_{j} .
$$

For high-order nonlinear function, the quadratic response surface is selected to approximate the real limit state function. The established response surface function is as follows:

$$
M=g(\mathbf{x})=a+\sum_{i=1}^{n} b_{i} x_{i}+\sum_{i=1}^{n} c_{i} x_{i}^{2} .
$$

In (11) and (12), $a, b_{j}, c_{i j}$, and $c_{i}$ are the undetermined coefficients.

3.3. Nonprobabilistic Response Surface Limit Method. The task of the nonprobabilistic response surface limit method is to solve the upper and lower values of the nonprobabilistic reliability index. Compared with the traditional nonprobabilistic response surface method, not only will the oscillating and nonconvergent phenomenon not appear in the iteration process of the nonprobabilistic response surface limit method, but also this iteration process is simpler and

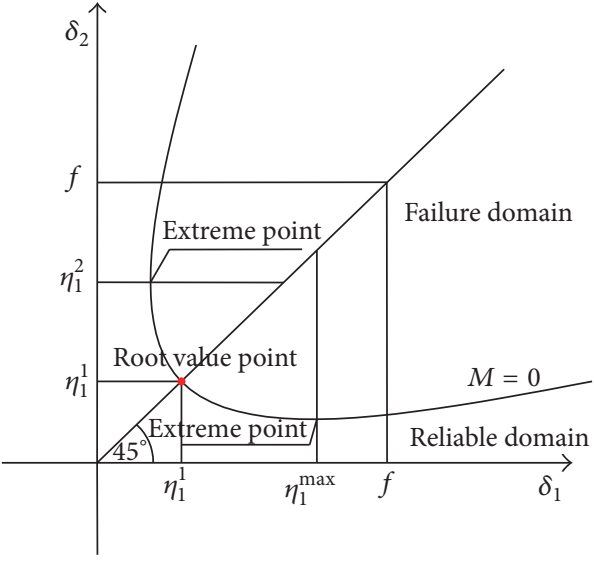

FIGURE 6: First iteration.

more rapid. The nonprobabilistic reliability index is finally obtained by iterating step by step. The basic steps are follows.

(1) Select the mean point as the initial point $\left(x_{1}{ }^{(0)}, x_{2}{ }^{(0)}\right.$, $\left.\ldots, x_{n}{ }^{(0)}\right)$ and $2 n$ points $\left(x_{1}{ }^{(0)}, \ldots, x_{i}{ }^{(0)} \pm f x_{i}{ }^{r}, \ldots, x_{n}{ }^{(0)}\right)$, where $x_{i}{ }^{(0)}$ and $x_{i}^{r}$ are the mid-value and dispersion of the interval variable $x_{i}(i=1,2, \ldots, n)$, respectively, and $f=2$.

(2) Based on the initial parameters, $(2 n+1)$ values for the function $M$ can be obtained through the finite element analysis and numerical simulation. Then $(2 n+1)$ undetermined coefficients $a, b_{i}, c_{i}(i=1,2, \ldots, n)$ can be obtained by solving the linear equation sets, so the initial response surface function $M=g(\mathbf{x})$ is known. Further, the regularized limit state function $G(\boldsymbol{\delta})$ will be obtained by standardizing the interval variable $x_{i}(i=1,2, \ldots, n)$.

(3) Solve all the nonprobabilistic reliability indices $\eta_{1}^{1}, \eta_{2}^{1}$, $\ldots, \eta_{n}^{1}$ using the Matlab software. Then obtain the maximum $\eta_{1}^{\max }$ which is not larger than the initial value of the parameter $f$ (if $\eta_{1}^{1}, \eta_{2}^{1}, \ldots, \eta_{n}^{1}$ are all larger than the initial value of the parameter $f$, the selected initial $f$ value is small and should increase the $f$ value). This process is also called the first iteration using the nonprobabilistic response surface limit method, as shown in Figure 6.

(4) Determine the new parameter points using the obtained $\eta_{1}^{\max }$ and then compute the nonprobabilistic reliability index until reaching the maximum $\eta_{2}^{\max }$, which is not larger than $\eta_{1}^{\max }$. This process is called the second iteration.

(5) Perform this iteration until the equation has no solution or the solution of the equation is not smaller than $\eta_{n-1}^{\max }$. Then the nonprobabilistic reliability index $\eta^{\min }$ (namely, $\eta_{n}^{\max }$ ) will be obtained. Actually here $\eta^{\min }$ is equal to $\eta_{n-1}^{\max }$. This process is called the $n$th iteration.

(6) Establish the new limit state function based on $\left[\eta^{\min }, \eta_{n-2}^{\max }\right]$. If and only if there exists $\eta_{n+1}$ or the condition of convergence can be satisfied, $\eta_{n+1}$ is the final value of the nonprobabilistic reliability index. The condition of convergence is as follows:

$$
\left|\eta_{n+1}-\eta_{n}^{\max }\right|<\varepsilon\left|\eta_{n}^{\max }\right| \quad(\varepsilon=0.01) .
$$

This final iteration process is also called the $(n+1)$ th iteration using the nonprobabilistic response surface limit method, as shown in Figure 7. 
TABLE 1: Comparison of calculation results.

\begin{tabular}{lccc}
\hline & Iterative steps & $\begin{array}{c}\text { Nonprobabilistic } \\
\text { reliability index }\end{array}$ & Relative error \\
\hline Analytical method & - & 1.7191 & - \\
Traditional nonprobabilistic response surface method & 5 & 1.7213 & $1.27 \times 10^{-3}$ \\
Presented nonprobabilistic response surface limit method & 2 & 1.7191 & 0 \\
\hline
\end{tabular}

Note. Relative error is obtained by comparing the nonprobabilistic reliability index calculated using other methods with that calculated using the analytical method.

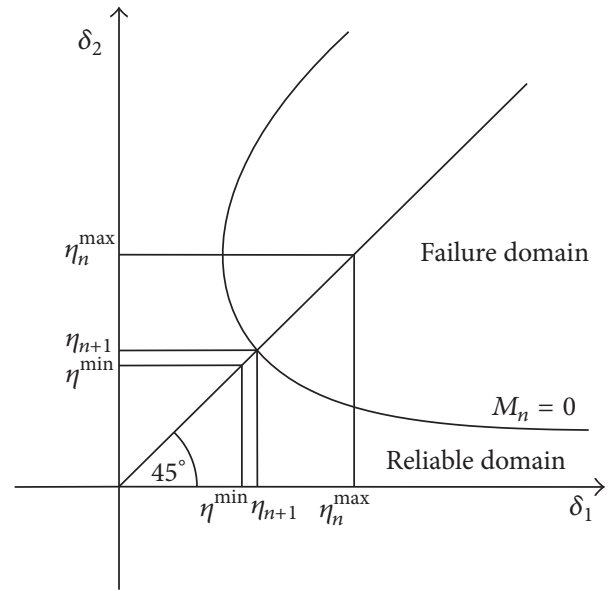

Figure 7: $(n+1)$ th iteration.

\section{Results and Discussion}

4.1. Example 1. Consider the following limit state function that includes three interval variables $x_{1}, x_{2}$, and $x_{3}$ :

$$
G=11 x_{1}^{2}+7 x_{2}^{2}-4 x_{3}^{2}+3,
$$

where $x_{1} \in[9,13], x_{2} \in[4,6]$, and $x_{3} \in[6.5,11.5]$.

Standardize the limit state function as shown by (14), so the performance function can be expressed as the following form again:

$$
\begin{aligned}
G= & 11\left(11+2 \times \delta_{1}\right)^{2}+7\left(5+1 \times \delta_{2}\right)^{2} \\
& -4\left(9+2.5 \times \delta_{3}\right)^{2}+3 .
\end{aligned}
$$

First, the global optimal solution method is used, and the nonprobabilistic reliability index can be computed; that is, $\eta=1.7191$. Next, the nonprobabilistic reliability index will be computed using the nonprobabilistic response surface limit method again. Letting $f=2$, after selecting the initial point, the fitted performance function can be given as follows:

$$
\begin{aligned}
G=3 & +2.65 \times 10^{-11} x_{1}+5.75 \times 10^{-11} x_{2}+1.57 \\
& \times 10^{-11} x_{3}+11 x_{1}^{2}+7 x_{2}^{2}-4 x_{3}^{2} .
\end{aligned}
$$

After the regularization of the above fitted performance function, the nonprobabilistic reliability indices are solved: $\eta=(-9.6727,-4.7119,2.2084,20.6377,1.7191,26.512)$. The maximum of the nonprobabilistic reliability indices, which are smaller than $f=2$, is 1.7191; that is, $\eta=1.7191$. Then letting $f=1.7191$, after the iterative computations, $\eta=1.7191$ can be computed again. This result satisfies the condition of convergence, so the nonprobabilistic reliability index is finally obtained: $\eta=1.7191$.

The nonprobabilistic reliability index computed using the traditional nonprobabilistic response surface method and that computed using the presented nonprobabilistic response surface limit method are summarized in Table 1.

4.2. Example 2. Consider the following limit state function which includes two interval variables $x_{1}$ and $x_{2}$ :

$$
G=x_{1}^{3}+x_{2}^{3}-4
$$

where $x_{1} \in[2,4]$ and $x_{2} \in[2.5,4.5]$.

Standardize the limit state function as shown by (17), so the performance function can be expressed as the following form again:

$$
G=\left(3+1 \times \delta_{1}\right)^{3}+\left(3.5+1 \times \delta_{2}\right)^{3}-4 .
$$

First, the Gradient Projection Method [19] is used, and the nonprobabilistic reliability index can be computed; that is, $\eta=2.0397$. Next, the nonprobabilistic reliability index will be computed using the presented nonprobabilistic response surface limit method again. After selecting the initial point, the computation process indicates that there is no solution with $f=2$. Then let $f=3$ again, and after four iterations the fitted performance function can be given as follows:

$$
\begin{aligned}
G= & -0.3196-2.57 x_{1}-6.103 \times 10^{-11} x_{2}+2.783 \\
& \times 10^{-11} x_{1}^{2} .
\end{aligned}
$$

With the above function, the results $\eta^{\text {min }}=1.962$ and $\eta^{\max }=2.178$ can be obtained. Meanwhile, the nonprobabilistic reliability indices can be also solved: $\eta=$ $(-2.5383,-2.7875,-3.339,-2.0397)$. Due to the condition of $\eta^{\min }<\eta<\eta^{\max }$, the nonprobabilistic reliability index is $\eta=2.0397$. Then letting $f=2.0397$, after the iterative computations, $\eta=2.0397$ can be computed again. This result satisfies the condition of convergence, so the nonprobabilistic reliability index is finally determined: $\eta=2.0397$.

The nonprobabilistic reliability index computed using the traditional nonprobabilistic response surface method and that computed using the nonprobabilistic response surface limit method are summarized in Table 2. 
TABLE 2: Comparison of calculation results.

\begin{tabular}{lccc}
\hline & Iterative steps & $\begin{array}{c}\text { Nonprobabilistic } \\
\text { reliability index }\end{array}$ & Relative error \\
\hline Analytical method & - & 2.0397 & - \\
Gradient Projection Method & - & 2.0397 & 0 \\
Traditional nonprobabilistic response surface method & 10 & 2.0396 & 2.0397 \\
Presented nonprobabilistic response surface limit method & 4 & 0 & 0 \\
\hline
\end{tabular}

Note. Relative error is obtained by comparing the nonprobabilistic reliability index calculated using other methods with that calculated using the analytical method.

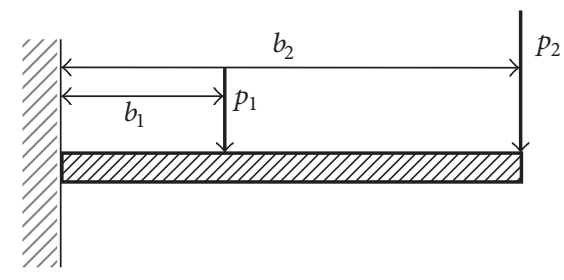

FIGURE 8: Schematic diagram of cantilever beam.

Table 2 indicates that, in solving the high nonlinear problems, the usage of the proposed nonprobabilistic response surface limit method will greatly reduce the iterative steps compared to the traditional nonprobabilistic response surface method. The computational efficiency is significantly improved when there is no difference in the solving accuracy.

4.3. Example 3. As shown in Figure 8, the concentrated loads $p_{1}$ and $p_{2}$ are applied on the cantilever beam, and the distances of these two concentrated loads from the fixed end are $b_{1}$ and $b_{2}$, respectively. Suppose the cantilever beam is failed when $\left|m_{\max }\right| \geq m_{\mathrm{cr}}$, where $m_{\mathrm{cr}}$ is the critical ultimate bending moment and $m_{\max }$ is the maximum of the bending moment of beam. The basic interval variables are $p_{1} \in[4.4$, $5.6] \mathrm{kN}, p_{2} \in[1.7,2.3] \mathrm{kN}, b_{1} \in[1.8,2.2] \mathrm{m}, b_{2} \in[4.5,5.5] \mathrm{m}$, and $m_{\mathrm{cr}} \in[32,40] \mathrm{kN} \cdot \mathrm{m}$. These parameters will be used in the estimation of the nonprobabilistic reliability for the structure.

Let $f=2$; the performance function is established using the quadratic form response surface:

$$
\begin{aligned}
G= & 20+x_{1}-2 x_{2}-5 x_{3}-5 x_{4}-2 x_{5}+6.046 \\
& \times 10^{-14} x_{1}^{2}+2.591 \times 10^{-12} x_{2}^{2}+9.266 \times 10^{-12} x_{3}^{2} \\
& +2.911 \times 10^{-11} x_{4}^{2}+3.187 \times 10^{-12} x_{5}^{2} .
\end{aligned}
$$

After the regularization of the performance function, the nonprobabilistic reliability indices are solved: $\eta=(2.807$, $12.308,3.721,22.857,4.324,-9.412,-3.404,-12.308,-3.721$, $-2.388,2.540,4.848,1.8391,-6.957,2.388)$. The maximum of the nonprobabilistic reliability indices, which are smaller than $f=2$, is 1.8391; that is, $\eta=1.8391$. Then letting $f=1.8391$, after the iterative computations, $\eta=1.8391$ can be computed again. This result satisfies the condition of convergence, so the nonprobabilistic reliability index is finally obtained: $\eta=1.8391$.
Let $f=2$; the performance function is established using the primary response surface:

$$
\begin{aligned}
G= & 1.1672+0.8836 x_{1}+1.7194 x_{2}-1.8054 x_{3} \\
& -1.0768 x_{4}+0.7384 x_{5}-0.0191 x_{1} x_{2} \\
& -0.0973 x_{1} x_{3}+0.2446 x_{1} x_{4}-0.0166 x_{1} x_{5} \\
& +1.0812 x_{2} x_{3}-3.3464 x_{2} x_{4}+0.2995 x_{2} x_{5} \\
& +2.0012 x_{3} x_{4}-1.8198 x_{3} x_{5}-1.837 \\
& \times 10^{-14} x_{4} x_{5} .
\end{aligned}
$$

After the regularization of the performance function, the nonprobabilistic reliability indices are solved: $\eta=(-5.423$, $24.616,-2.414,-2.319,5.003,6.51,-9.103,2.421,-33.967$, $1.7446,-7.755,20.966,-220.919,-8.065,2.546,4.132,5.657$, $-11.984,2.274,-51.899)$. The maximum of the nonprobabilistic reliability indices, which are smaller than $f=2$, is 1.7446; that is, $\eta=1.7446$. Then letting $f=1.7446$, after the iterative computations, $\eta=1.7446$ can be computed. These results satisfy the condition of convergence, so the nonprobabilistic reliability index is finally obtained: $\eta=1.7446$.

The nonprobabilistic reliability index computed using the one-dimensional optimization algorithm $[15,16]$ is $\eta=1.7446$. Finally, the nonprobabilistic reliability indices, computed using the one-dimensional optimization algorithm, the quadratic form response surface method, and the primary response surface method, are summarized in Table 3.

Table 3 indicates that the forms of the response surface functions have great influence on the final nonprobabilistic reliability index. The response surface function built greatly approximates the real limit state function, and the nonprobabilistic reliability index obtained greatly approximates the real value.

4.4. Example 4. A PC (prestressed reinforced concrete) continuous rigid frame bridge with three spans is investigated. Three spans are $75 m+130 m+75 m$, respectively, and the substructure is twin thin-wall pier.

4.4.1. Nonprobabilistic Reliability Evaluation Process. The proposed nonprobabilistic response surface limit method will be used to evaluate the bridge reliability. The measured data is obtained for building the Finite Element Model (FEM). The nonprobabilistic reliability index is solved based on 
TABLE 3: Comparison of calculation results.

\begin{tabular}{lccc}
\hline & Iterative steps & $\begin{array}{c}\text { Nonprobabilistic } \\
\text { reliability index }\end{array}$ & Relative error \\
\hline Analytical method & - & 1.7446 & - \\
One-dimensional optimization algorithm & - & 1.7446 & 0 \\
Quadratic form response surface method & 2 & 1.8391 & 1.7446 \\
Primary response surface method & 2 & $5.4 \times 10^{-2}$ \\
\hline
\end{tabular}

Note. Relative error is obtained by comparing the nonprobabilistic reliability index calculated using other methods with that calculated using the analytical method.

TABLE 4: Initial value (before reinforcement).

\begin{tabular}{|c|c|c|c|c|}
\hline Compressive strength $(\mathrm{MPa})$ & Area of the prestressed tendon $(\mathrm{mm})$ & $M=R-S(\mathrm{kN} \cdot \mathrm{m})$ & $R(\mathrm{kN} \cdot \mathrm{m})$ & $S(\mathrm{kN} \cdot \mathrm{m})$ \\
\hline 45 & 140 & 4294.3 & 175427.3 & 179721.6 \\
\hline 40 & 140 & 12996.5 & 165715.9 & 178712.4 \\
\hline 50 & 140 & -1072.5 & 181548.0 & 180475.5 \\
\hline 45 & 130 & -1784.9 & 169259.3 & 167474.3 \\
\hline 45 & 150 & 10282.6 & 181596.3 & 191878.9 \\
\hline
\end{tabular}

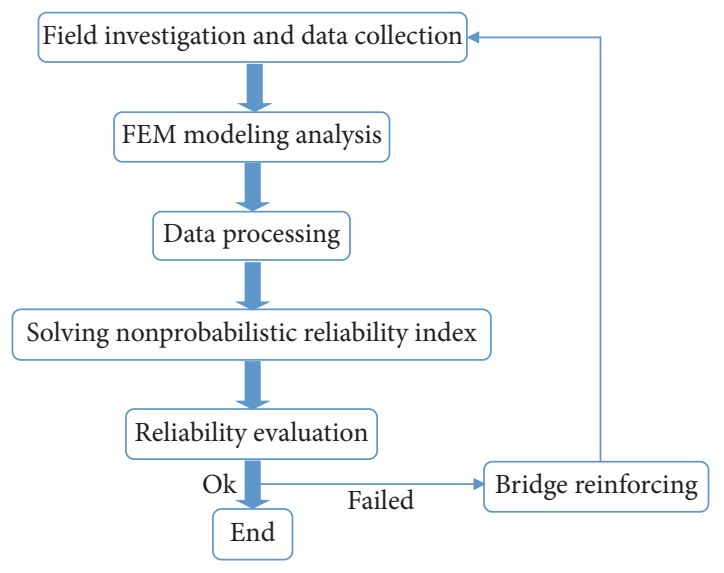

FIGURE 9: Reliability evaluation process.

the processed data using the Matlab software. The specific evaluation process is shown in Figure 9.

4.4.2. Finite Element Model. The measured relative parameters are as follows: height of main beam $h \in[295,305] \mathrm{cm}$; bridge deck width $B \in[1645,1655] \mathrm{cm}$; reinforced concrete weight $\gamma \in[23,25] \mathrm{kN} / \mathrm{m}^{3}$; concrete compressive strength $C \in[40,50] \mathrm{MPa}$; area of the single prestressed tendon $A \in$ $[135,145] \mathrm{mm}^{2}$.

The sensitivity analysis indicates that concrete compressive strength and area of the single prestressed tendon have great influence on the bending moment. Therefore, the reliability analysis will be conducted by selecting concrete compressive strength $C$ and area of the single prestressed tendon $A$ as the variables. The Finite Element Model is established using the Midas software, as shown in Figure 10. The maximum resistance and effect values of the control section will be also computed using the Midas software. The nonprobabilistic reliability analysis of the structure will be

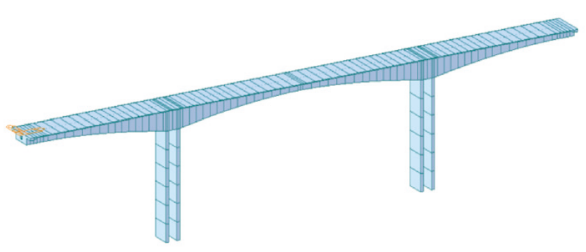

Figure 10: Finite Element Model.

performed by selecting the midspan section as the control section.

4.4.3. Reliability Evaluation before Reinforcement. Let $f=2$; the initial parameters of the model, the resistance $(R)$, and the effect $(S)$ computed using the Midas software are summarized in Table 4.

Now the proposed nonprobabilistic response surface limit method is utilized to solve the nonprobabilistic reliability index based on the parameters in Table 4 . The fitted performance function can be given as follows:

$$
\begin{aligned}
G= & 1.09 \times 10^{5}-7.41 \times 10^{3} x_{1}+730.7 x_{2}+66.7 x_{1}^{2} \\
& -0.45 x_{2}^{2} .
\end{aligned}
$$

After the regularization of the performance function, the nonprobabilistic reliability indices are solved: $\eta=$ $(4.218,132.733,15.425,0.686)$. The maximum of the nonprobabilistic reliability indices, which are smaller than $f=$ 2 , is 0.686 ; that is, $\eta=0.686$. Then letting $f=0.686$, after the first iterative computations, the iterative results are summarized in Table 5.

Next the proposed nonprobabilistic response surface limit method is again utilized to solve the nonprobabilistic reliability index based on the parameters in Table 5. The fitted performance function can be given as follows: 
TABLE 5: First iterative computation results (before reinforcement).

\begin{tabular}{|c|c|c|c|c|}
\hline Compressive strength (MPa) & Area of the prestressed tendon $(\mathrm{mm})$ & $M=R-S(\mathrm{kN} \cdot \mathrm{m})$ & $R(\mathrm{kN} \cdot \mathrm{m})$ & $S(\mathrm{kN} \cdot \mathrm{m})$ \\
\hline 45 & 140 & 4294.3 & 175427.3 & 179721.6 \\
\hline 43.29 & 140 & 7042.5 & 171665.5 & 178708.1 \\
\hline 46.72 & 140 & 1679.4 & 178796.7 & 180476.1 \\
\hline 45 & 136.57 & 2214.5 & 173313.5 & 175528.0 \\
\hline 45 & 143.43 & 6363.3 & 177541.4 & 183904.7 \\
\hline
\end{tabular}

TABLE 6: Second iterative computation results (before reinforcement).

\begin{tabular}{lcccc}
\hline Compressive strength $(\mathrm{MPa})$ & Area of the prestressed tendon $(\mathrm{mm})$ & $M=R-S(\mathrm{kN} \cdot \mathrm{m})$ & $R(\mathrm{kN} \cdot \mathrm{m})$ & $S(\mathrm{kN} \cdot \mathrm{m})$ \\
\hline 45 & 140 & 4294.3 & 175427.3 & 179721.6 \\
43.43 & 140 & 6838.5 & 171869.4 & 178707.9 \\
46.57 & 140 & 1829.7 & 178646.4 \\
45 & 136.86 & 2403.5 & 173501.7 \\
45 & 143.14 & 6199.6 & 175476.1 \\
\hline
\end{tabular}

TABLE 7: Third iterative computation results (before reinforcement).

\begin{tabular}{|c|c|c|c|c|}
\hline Compressive strength (MPa) & Area of the prestressed tendon $(\mathrm{mm})$ & $M=R-S(\mathrm{kN} \cdot \mathrm{m})$ & $R(\mathrm{kN} \cdot \mathrm{m})$ & $S(\mathrm{kN} \cdot \mathrm{m})$ \\
\hline 45 & 140 & 4294.3 & 175427.3 & 179721.6 \\
\hline 43.45 & 140 & 6809.7 & 171898.2 & 178707.9 \\
\hline 46.55 & 140 & 1849.9 & 178626.2 & 180476.1 \\
\hline 45 & 136.91 & 2437.0 & 173518.8 & 175955.8 \\
\hline 45 & 143.1 & 6166.3 & 177349.3 & 183515.7 \\
\hline
\end{tabular}

$$
\begin{aligned}
G= & 3.22 \times 10^{4}-3.84 \times 10^{3} x_{1}+733.8 x_{2}+25.3 x_{1}^{2} \\
& -0.46 x_{2}^{2} .
\end{aligned}
$$

After the regularization of the performance function as shown by (23), the nonprobabilistic reliability indices are obtained: $\eta=(12.36,131.24,46.64,0.628)$. The maximum of the nonprobabilistic reliability indices, which are smaller than $f=0.686$, is 0.628 ; that is, $\eta=0.628$. Then letting $f=$ 0.628 , after the second iterative computations, the iterative results are summarized in Table 6.

Next the presented nonprobabilistic response surface limit method is again utilized to solve the nonprobabilistic reliability index based on the parameters in Table 6 . The fitted performance function can be given as follows:

$$
\begin{aligned}
G= & 3.85 \times 10^{4}-3.05 \times 10^{3} x_{1}+398.6 x_{2}+16.1 x_{1}^{2} \\
& +0.74 x_{2}^{2} .
\end{aligned}
$$

After the regularization of the performance function as shown by (24), the nonprobabilistic reliability indices are computed: $\eta=(19.768,-82.202,58.167,0.619)$. The maximum of the nonprobabilistic reliability indices, which are smaller than $f=0.628$, is 0.619 ; that is, $\eta=0.619$. Then let $f=0.619$, after the third iterative computations, the iterative results are summarized in Table 7.

Next the proposed nonprobabilistic response surface limit method is again utilized to solve the nonprobabilistic reliability index based on the parameters in Table 7 . The fitted performance function can be given as follows:

$$
\begin{aligned}
G= & 3.07 \times 10^{4}-2.93 \times 10^{3} x_{1}+476 x_{2}+14.8 x_{1}^{2} \\
& -0.45 x_{2}^{2} .
\end{aligned}
$$

After the regularization of the performance function as shown by (25), the nonprobabilistic reliability indices are computed: $\eta=(21.680,-133.413,67.108,0.618)$. The maximum of the nonprobabilistic reliability indices, which are smaller than $f=0.619$, is 0.618 ; that is, $\eta=0.618$. Here $(0.619-0.618) / 0.618=0.0016<0.01$, which satisfies the condition of convergence. In summary, the nonprobabilistic reliability index is finally determined, which is $\eta=0.618$.

4.4.4. Reliability Evaluation after Reinforcement. According to $\eta=0.618<1$, the bridge is unreliable. Then 16 bundles of the external prestressed tendon are adopted to reinforce the unreliable bridge, and the nonprobabilistic reliability index for the reinforced bridge is solved again.

Let $f=2$; the initial parameters of the model, the resistance $(R)$, and the effect $(S)$ computed using the Midas software are summarized in Table 8.

Next the proposed nonprobabilistic response surface limit method is again utilized to solve the nonprobabilistic reliability index based on the parameters in Table 8 . The fitted performance function can be given as follows:

$$
\begin{aligned}
G= & 1.15 \times 10^{5}-7.62 \times 10^{3} x_{1}+783.1 x_{2}+68.7 x_{1}^{2} \\
& -0.49 x_{2}^{2} .
\end{aligned}
$$


TABLE 8: Initial value (after reinforcement).

\begin{tabular}{|c|c|c|c|c|}
\hline Compressive strength (MPa) & Area of the prestressed tendon $(\mathrm{mm})$ & $M=R-S(\mathrm{kN} \cdot \mathrm{m})$ & $R(\mathrm{kN} \cdot \mathrm{m})$ & $S(\mathrm{kN} \cdot \mathrm{m})$ \\
\hline 45 & 140 & 10879.6 & 179122.2 & 190001.8 \\
\hline 40 & 140 & 19787.6 & 169093.5 & 188881.1 \\
\hline 50 & 140 & 5404.2 & 185434.4 & 190838.6 \\
\hline 45 & 130 & 4377.0 & 172708.7 & 177085.7 \\
\hline 45 & 150 & 17283.8 & 185534.1 & 202817.9 \\
\hline
\end{tabular}

TABLE 9: First iterative computation results (after reinforcement).

\begin{tabular}{|c|c|c|c|c|}
\hline Compressive strength $(\mathrm{MPa})$ & Area of the prestressed tendon $(\mathrm{mm})$ & $M=R-S(\mathrm{kN} \cdot \mathrm{m})$ & $R(\mathrm{kN} \cdot \mathrm{m})$ & $S(\mathrm{kN} \cdot \mathrm{m})$ \\
\hline 45 & 140 & 10879.6 & 179122.2 & 190001.8 \\
\hline 40.52 & 140 & 18633.3 & 170246.9 & 188880.2 \\
\hline 49.48 & 140 & 5796.5 & 185042.3 & 190838.8 \\
\hline 45 & 131.05 & 5053.5 & 173378.8 & 178432.3 \\
\hline 45 & 148.96 & 16625.6 & 184865.4 & 201490.9 \\
\hline
\end{tabular}

After the regularization of the performance function as shown by (26), the nonprobabilistic reliability indices are computed: $\eta=(4.19,131.191,14.579,1.791)$. The maximum of the nonprobabilistic reliability indices, which are smaller than $f=2$, is 1.791 ; that is, $\eta=1.791$. Then letting $f=1.791$, after the first iterative computations, the iterative results are summarized in Table 9.

Next the proposed nonprobabilistic response surface limit method is again utilized to solve the nonprobabilistic reliability index based on the parameters in Table 9. The fitted performance function can be given as follows:

$$
\begin{aligned}
G= & 1.09 \times 10^{5}-7.42 \times 10^{3} x_{1}+797.3 x_{2}+66.5 x_{1}^{2} \\
& -0.54 x_{2}^{2} .
\end{aligned}
$$

After the regularization of the performance function as shown by (27), the nonprobabilistic reliability indices are computed: $\eta=(4.307,119.671,15.147,1.785)$. The maximum of the nonprobabilistic reliability indices, which are smaller than $f=1.791$, is 1.785 ; that is, $\eta=1.785$. Here $(1.791-1.785) / 1.785=0.0034<0.01$ satisfies the condition of convergence. In summary, the nonprobabilistic reliability index is finally determined; that is, $\eta=1.785$, which indicates that the bridge is reliable.

\section{Conclusions}

This article is the first to successfully propose a nonprobabilistic response surface limit method to perform nonprobabilistic reliability analysis for the structures based on the interval model. Using this method, nonprobabilistic reliability analysis can be performed with the conditions of unknown performance function and data shortage. Compared with the traditional nonprobabilistic response surface method, there are two other main advantages of the proposed method: (1) it effectively avoids the tedious and oscillating iteration process; (2) it greatly enhances computational efficiency and precision and obtains the nonprobabilistic reliability index rapidly. The complete nonprobabilistic reliability evaluation process for the bridge was illustrated and built through evaluating the reliability of one PC continuous rigid frame bridge with three spans. The results indicated that the reliability level of the selected PC continuous rigid frame bridge with three spans was very low, so that reinforcement measures were needed to improve the performance of this bridge. The further reliability estimation for the reinforced bridge demonstrated that the reliability level of the reinforced bridge was greatly improved, and the reinforcements were effective. In summary, this study illustrated the effectiveness and feasibility of the proposed nonprobabilistic response surface limit method in the bridge nonprobabilistic reliability estimation through theoretical analysis and example verification.

\section{Conflicts of Interest}

The authors declare that they have no conflicts of interest.

\section{Acknowledgments}

The authors would like to express their gratitude to the National Natural Science Foundation of China (Grants 51408444 and 51708428).

\section{References}

[1] J. Song and Z.-Z. Lv, "New reliability sensitivity analysis method," Acta Aeronautica et Astronautica Sinica, vol. 27, no. 5, pp. 823-826, 2006.

[2] I. Elishakoff, "Essay on uncertainties in elastic and viscoelastic structures: from A. M. Freudenthal's criticisms to modern convex modeling," Computers \& Structures, vol. 56, no. 6, pp. 871-895, 1995.

[3] Y. Ben-Haim, "Convex models of uncertainty in radial pulse buckling of shells," Transactions of ASME: Journal of Applied Mechanics, vol. 60, no. 3, pp. 683-688, 1993.

[4] Y. Ben-Haim, "A non-probabilistic concept of reliability," Structural Safety, vol. 14, no. 4, pp. 227-245, 1994. 
[5] Y. Ben-Haim, "A non-probabilistic measure of reliability of linear systems based on expansion of convex models," Structural Safety, vol. 17, no. 2, pp. 91-109, 1995.

[6] Y. Ben-Haim and I. Elishakoff, "Discussion on: a nonprobabilistic concept of reliability," Structural Safety, vol. 17, no. 3, pp. 195-199, 1995.

[7] S. X. Guo, Z. Z. Lv, and Y. S. Feng, "A non-probabilistic model of structural reliability based on interval analysis," Chinese Journal of Computational Mechanics, vol. 18, pp. 56-62, 2001.

[8] S. X. Guo and Z. Z. Lv, "Comparison between the nonprobabilistic and probabilistic reliability methods for uncertain structure design," Chinese Journal of Applied Mechanics, vol. 20, pp. 107-110, 2003.

[9] Z. P. Qiu, Y. Y. Xia, and J. L. Yang, “The static displacement and the stress analysis of structures with bounded uncertainties using the vertex solution theorem," Computer Methods Applied Mechanics and Engineering, vol. 196, no. 49-52, pp. 4965-4984, 2007.

[10] Z. P. Qiu and J. Wang, "The interval estimation of reliability for probabilistic and non-probabilistic hybrid structural system," Engineering Failure Analysis, vol. 17, no. 5, pp. 1142-1154, 2010.

[11] Z. P. Qiu, P. C. Mueller, and A. Frommer, "The new nonprobabilistic criterion of failure for dynamical systems based on convex models," Mathematical and Computer Modelling, vol. 40, no. 1-2, pp. 201-215, 2004.

[12] C. Jiang, R. G. Bi, G. Y. Lu, and X. Han, "Structural reliability analysis using non-probabilistic convex model," Computer Methods Applied Mechanics and Engineering, vol. 254, pp. 8398, 2013.

[13] C. Jiang, Q. F. Zhang, X. Han, and Y. H. Qian, "A nonprobabilistic structural reliability analysis method based on a multidimensional parallelepiped convex model," Acta Mechanica, vol. 225, no. 2, pp. 383-395, 2014.

[14] Y.-J. Luo and Z. Kang, "Iteration approach for structural non-probabilistic reliability analysis based on hyper-ellipsoidal models," Chinese Journal of Computational Mechanics, vol. 25, no. 6, pp. 747-752, 2008.

[15] T. Jiang, J. J. Chen, P. G. Jiang et al., "A one-dimensional optimization algorithm for non-probabilistic reliability index," Engineering Mechanics, vol. 24, pp. 23-27, 2007.

[16] X. Chen, C.-Y. Tang, C.-P. Tsui, and J. Fan, "Modified scheme based on semi-analytic approach for computing nonprobabilistic reliability index," Acta Mechanica Solida Sinica, vol. 23, no. 2, pp. 115-123, 2010.

[17] J. P. Fan, S. J. Li, and X. Y. Chen, "Optimal searching algorithm for non-probabilistic reliability," Chinese Journal of Computational Mechanics, vol. 29, pp. 831-834, 2012.

[18] C. Jiang, Z. Zhang, X. Han et al., "An evidence-theory-based reliability analysis method for uncertain structures," Chinese Journal of Theoretical and Applied Mechanics, vol. 45, pp. 103115, 2013.

[19] S.-J. Li, J.-P. Fan, W. Qi, and X.-Y. Chen, "The gradient projection method for non-probabilistic reliability index based on interval model," Chinese Journal of Computational Mechanics, vol. 30, no. 2, pp. 192-197, 2013.

[20] X. Chen, J. Fan, and X. Bian, "Structural robust optimization design based on convex model," Results in Physics, vol. 7, pp. 3068-3077, 2017.

[21] Y. Liu, H. K. Jeong, and M. Collette, "Efficient optimization of reliability-constrained structural design problems including interval uncertainty," Computers \& Structures, vol. 177, pp. 1-11, 2016.
[22] T. Jiang, J. J. Chen, J. G. Zhang et al., "Response surface method based on interval models and interval analysis," Machine Design and Research, vol. 21, pp. 12-16, 2005.

[23] J. Y. Chen, W. C. Wen, and Y. J. Wang, "Analysis of structural non-probabilistic reliability based on response surface method," Journal of Zhengzhou University (Engineering Science), vol. 36, pp. 121-124, 2015.

[24] J. Tao, C. Jian-Jun, and X. Ya-Lan, "A semi-analytic method for calculating non-probabilistic reliability index based on interval models," Applied Mathematical Modelling, vol. 31, no. 7, pp. 1362-1370, 2007.

[25] J. P. Fan and X. Y. Chen, "Non-probabilistic reliability global optimal solution based on interval model," Journal of Computational Mechanics, vol. 35, pp. 476-484, 2014.

[26] G. E. P. Box and K. B. Wilson, "On the experimental attainment of optimum conditions," Journal of the Royal Statistical Society: Series B (Statistical Methodology), vol. 13, no. 1, pp. 1-45, 1951.

[27] R. H. Myers and D. C. Montgomery, Response Surface Methodology, John Wiley \& Sons, Inc., New York, NY, USA, 1995. 


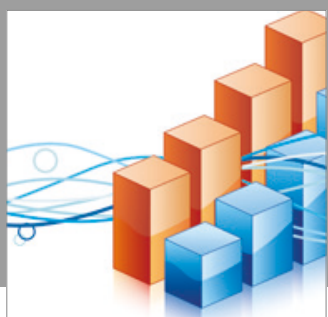

Advances in

Operations Research

vatersals

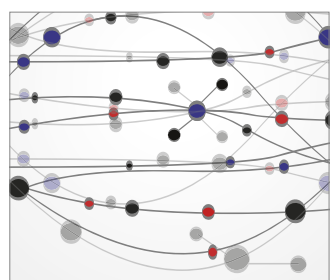

\section{The Scientific} World Journal
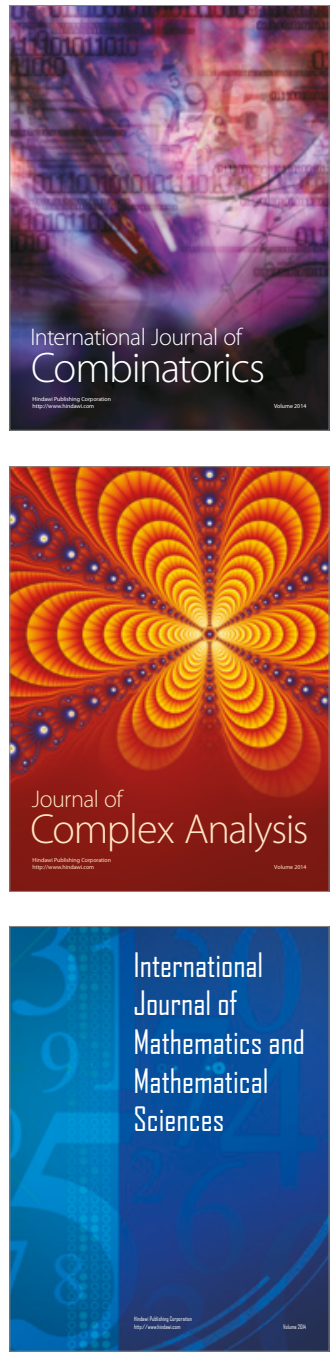
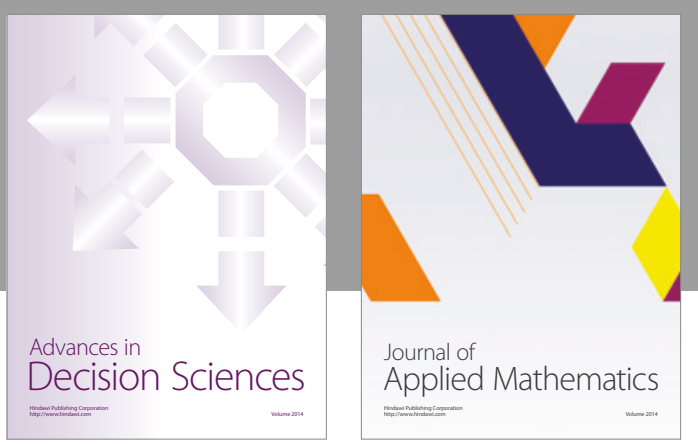

Algebra

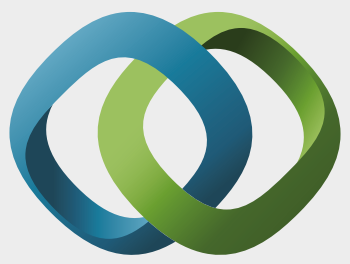

\section{Hindawi}

Submit your manuscripts at

https://www.hindawi.com
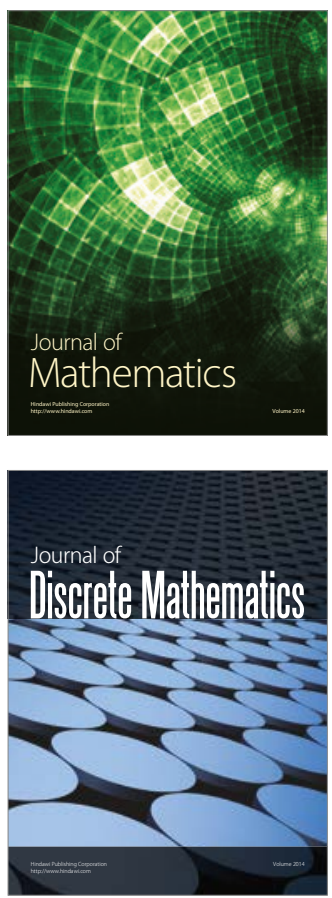

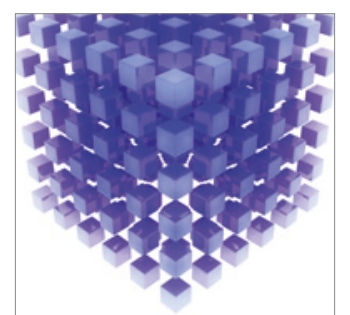

Mathematical Problems in Engineering
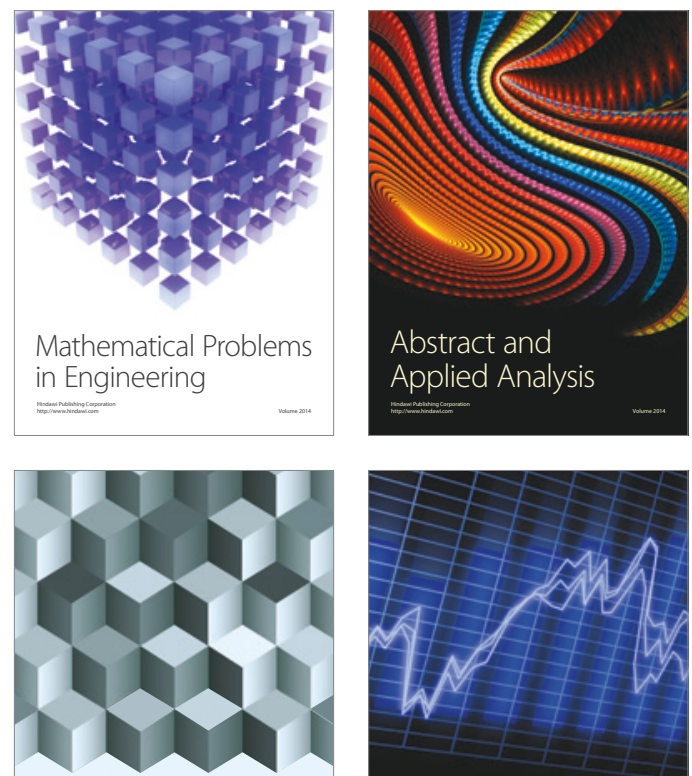

Journal of

Function Spaces

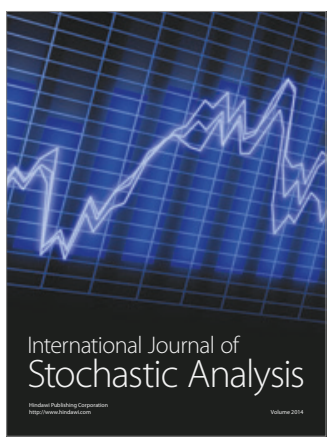

Probability and Statistics
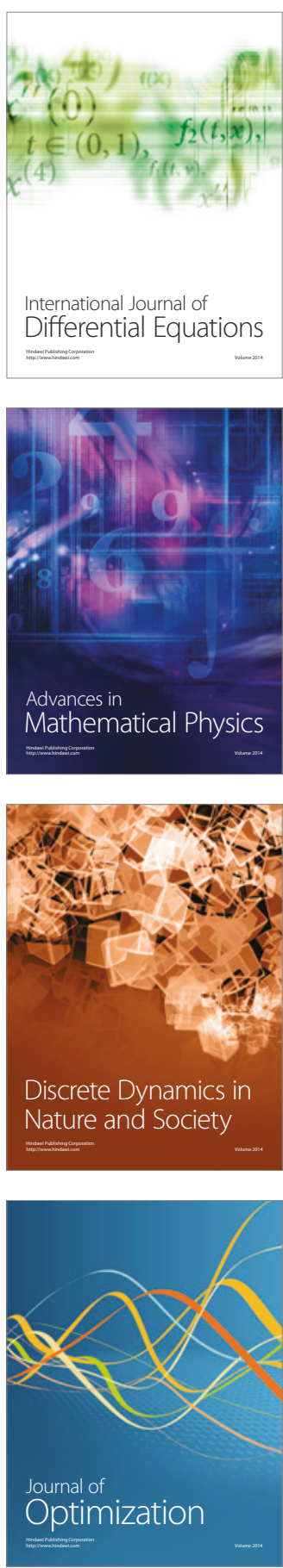